\title{
Volante de inercia: sistemas y materiales aplicados en medios de transporte de pasajeros
}

FESS Technology: Systems and Materials Applied in Passenger Transport

\begin{tabular}{c} 
Remache A. \\
Instituto Superior Tecnológico Kachariy. Quito, Ecuador \\
e-mail: abel.remache@itk.edu.ec \\
\hline Información del artículo \\
Recibido: febrero 2020 \\
Aceptado: mayo 2020 \\
\hline
\end{tabular}

\begin{abstract}
RESUMEN
Se ha realizado un estudio del uso de la energía cinética a través de la tecnología de los volantes de inercia FESS. Se detalla el principio de funcionamiento, su estructura interna, configuraciones y diversas consideraciones técnicas que fueron necesarias para su implementación en varias aplicaciones en la actualidad. Se identifican los usos en medios de transporte de sistemas KERS, el uso de tecnología de levitación a través de los HTS, aplicaciones hibridas y sistemas Gimbal entre otros. Se define el uso de materiales ya que es primordial para su capacidad energética y costo final en el desarrollo de esta tecnología. Para finalizar se analiza el potencial que tiene un volante de inercia y las limitaciones a las que se enfrenta en el ámbito de construcción, costos, materiales y densidad energética. Se concluye que los volantes se han integrado a aplicaciones sencillas y avanzadas, con ventajas tales como un costo ambiental bajo, larga vida útil, bajo mantenimiento y poca fricción, reducción de emisiones, entre otras. Entre las desventajas están la baja densidad de energía, costos, uso de materiales poco accesibles, peso, entre otros; por ende, la investigación actual trabaja en estos campos.
\end{abstract}

Palabras clave: energía cinética, volantes de inercia FESS, transporte, materiales

\begin{abstract}
A study of the use of kinetic energy through the technology of FESS inertia wheels has been carried out. It details the operation principle, its internal structure, configurations and various technical considerations that were necessary for its implementation in several applications at present. It identifies the uses in means of transport of KERS systems, the use of levitation technology through the HTS, hybrid applications and Gimbal systems among others. The use of materials is defined as it is essential for their energy capacity and final cost in the development of this technology. Finally, the potential of a flywheel and the limitations it faces in the field of construction, costs, materials and energy density are analyzed. It is concluded that the flyers have been integrated into simple and advanced applications, with advantages such as a low environmental cost, long service life, low maintenance and low friction, reduction of emissions, among others. Among the disadvantages are the low energy density, costs, use of materials that are not very accessible, weight, among others; therefore, current research works in these fields.
\end{abstract}

Keywords: kinetic energy, FESS technology, transportation, materials 


\section{Introducción}

Según Schutlz (2014), las áreas de importancia en el uso vigente del volante inercial está relacionado con: Industria automotriz, Fuentes de alimentación ininterrumpibles, Militar / Defensa, Almacenamiento de energía de red, Aerogeneradores [1].

Diversas aplicaciones en volantes de inercia han generado buenos resultados como por ejemplo volantes de inercia con una capacidad de energía específica del rotor de $195 \mathrm{Wh} / \mathrm{kg}$, comparable con las baterías de iones de litio. [2].

Otras aplicaciones van desde la gestión de energía conectada a la red hasta el lanzamiento de aeronaves electromagnéticas. Las configuraciones de rotor predominantes están estructuradas mediante discos, cilindros sólidos y cilindros de pared gruesa fabricados en carbono y compuesto de vidrio o acero de alta resistencia[3].

Este estudio se concentra en aplicaciones relacionadas a los medios de transporte, en las que los volantes son utilizados en el almacenamiento y ahorro significativo de energía en sistemas automotrices. Mencionando un ejemplo, vehículos de transporte de pasajeros como automóviles y trenes tienen este sistema y varios son capaces de ahorrar hasta un $15 \%$ de combustible en conducción por ciudad [4], siendo accionado en frenadas y paradas, en varios casos dependiendo de su sistema el motor de combustión se detiene y, así, se ahorra el consumo innecesario del combustible que produce gases contaminantes y cuyo costo es elevado en la actualidad.

\section{Desarrollo}

\subsection{Funcionamiento ygeneralidades}

Un volante de Inercia es un dispositivo mecánico giratorio utilizado para almacenar energía cinética rotatoria. Los volantes tienen un momento significativo de inercia y por ende resisten variaciones en la velocidad de rotación. La cantidad de energía almacenada en un volante es proporcional al cuadrado de su velocidad de rotación (Ver ecuación 1). La energía se transfiere a un volante a través de la aplicación de par externo, incrementando así su velocidad de rotación, y por lo tanto su energía almacenada. Un volante libera su energía almace- nada aplicando un par a una carga mecánica, de esta forma se reduce su rotación. [5]

En la actualidad y generalmente, el volante de inercia está dentro de un contenedor al vacío para eliminar la pérdida de fricción causada por el aire y suspendido por cojinetes para una operación estable. La energía cinética se transfiere dentro y fuera del volante pudiendo funcionar como motor o como generador dependiendo del ángulo de carga. Al actuar como motor, la energía eléctrica suministrada se convierte en par y se aplica al rotor; en el modo generador, la energía cinética almacenada en el rotor aplica un par, que se convierte en energía eléctrica. Aparte del volante se requiere una electrónica de potencia adicional para controlar la entrada y salida de potencia, la velocidad, la frecuencia, entre otras [6].

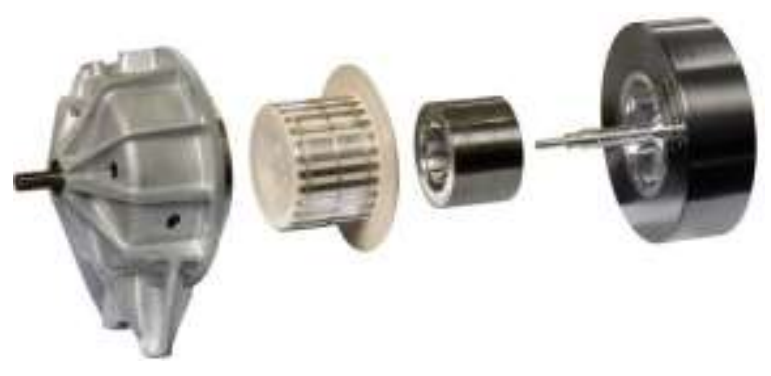

Figura 1. Estructura del volante de inercia [7].

Formulaciones básicas. - Como se ha mencionado, la energía cinética almacenada en un volante es proporcional a la masa y al cuadrado de su velocidad de rotación acorde con la Ecuación (1).

$$
E_{k}=\frac{1}{2} I w^{2}
$$

Donde: $E_{k}=$ Energía cinética almacenada en el volante (Kinetic energy),

$\mathrm{I}=$ Momento de inercia,

$w=$ Velocidad angular del volante,

Para los rotores de acero (volantes), la forma dominante es un cilindro sólido, por lo que se da la siguiente expresión para I:

$$
I=\frac{1}{2} r^{2} m=\frac{1}{2} r^{4} \pi a \rho
$$


Donde: $r=$ radio,

$a=$ longitud del cilindro,

$m=$ masa del cilindro,

$\rho=$ densidad del material del cilindro,

Cuando la forma supone un cilindro circular hueco, conduce a la ecuación 3

$$
I=\frac{1}{4} m\left(r_{0}{ }^{2}-r_{i}{ }^{2}\right)=\frac{1}{2} \pi a \rho\left(r_{0}{ }^{4}-r_{i}^{4}\right)
$$

Donde: $r_{0}=$ radio externo,

$$
\mathrm{r}_{\mathrm{i}}=\text { radio interno }
$$

Así la manera más eficiente de aumentar la energía almacenada es acelerar el volante. El límite de velocidad se logra a través de cargas inerciales o resistencia a la tracción. Los materiales más ligeros desarrollan cargas de inercia más bajas a una velocidad dada, por lo tanto los materiales compuestos, con baja densidad y alta resistencia a la tracción, son excelentes para almacenar energía cinética [8]. De esta manera la energía cinética por unidad de volumen y masa se define por [9]:

$e_{v}=K \sigma$

em $=K \sigma / \rho$

Donde:

$\mathrm{e}_{\mathrm{v}}=$ energía cinética por unidad de volumen, $\mathrm{e}_{\mathrm{m}}=$ energía cinética por unidad de masa,

$K=$ es el factor de forma,

$\sigma=$ tensión máxima en el volante.

Las tensiones en un volante de inercia influirán en las tres dimensiones. Para un rotor construido con un material no isotrópico, como compuestos con fibra, la interacción de esfuerzos limitará las dimensiones prácticas posibles. En diseños cortos (generalmente volantes de inercia huecos), las tensiones de interés primario son la tensión radial $\sigma_{\Gamma}$ (Ecuación 6) y la tensión del aro $\sigma \theta$ (Ecuación 7) [8]. Para un material isotrópico, la tensión radial y de aro se expresan mediante las ecuaciones:

$\sigma_{\Gamma}=\frac{3+v}{8} \rho w^{2}\left(r_{0}^{2}+r_{i}^{2}-\frac{r_{0}^{2} r_{i}^{2}}{r^{2}}-r^{2}\right)$

Donde: $v=$ coeficiente de Poisson,

$r=$ cualquier radio dentro del rotor.

$$
\sigma_{\theta}=\frac{3+v}{8} \rho w^{2}\left(r_{0}^{2}+r_{i}^{2}+\frac{r_{0}^{2} r_{i}^{2}}{r^{2}}-\frac{1+3 v}{3+v} r^{2}\right)
$$

Varios datos de materiales para volantes de inercia son posibles encontrar en tablas como información abierta, así como los factores de forma $K$

\subsection{Transporte liviano}

\subsubsection{Sistema KERS}

El sistema KERS (Kinetic Energy Recovery System), implementado en vehículos de turismo, se basa en los principios de funcionamiento del volante de inercia, puede actuar como dinamo-alternador en fases de frenada, y como motor eléctrico de corriente continua en fases de aceleración. Peroa diferencia de la versión mecánica, el KERS eléctrico puede tener o no baterías químicas para almacenar energía (Figura 2). Flybrid (empresa desarrolladora KERS) junto a Magnetti Marelli desarrolló un dispositivo híbrido entre el eléctrico y el mecánico de $27 \mathrm{~kg}$; esta versión del KERS almacena en una cámara de vacío, un volante de inercia que gira libre de rozamientos gracias a la energía cinética recuperada en las frenadas, que puede producir el giro de un rotor de reluctancia variable sobre un estator, generando corriente alterna que rectificada a continua (ac-dc), alimenta a un motor eléctrico [10].

El KERS ha desarrollado un sistema de vacío, generando un estado de giro en extremo duradero, debido a la inercia generada, que solo se pierde en fases de aceleración cuando la energía cinética se aplica a la tracción del vehículo. El sistema KERS mecánico no transforma la energía cinética en eléctrica, solo utiliza su movimiento para ayuda de tracción, y por tanto no necesita ningún tipo de motor eléctrico o batería[10].

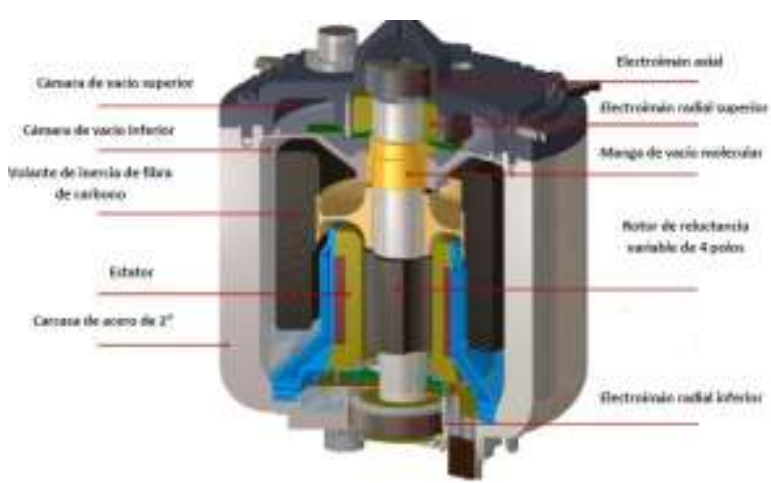

Figura 2. Volante de inercia KERS [10] 
Los sistemas de recuperación de energía cinética no obtuvieron los resultados esperados en la Fórmula 1, pero varias compañías automotrices insertan e investigan esta tecnología en automóviles de turismo. Varias aplicaciones se han dado en los últimos años combinando esta tecnología con la transmisión CVT (Continuously Variable Transmission), para mejorar los resultados en automóviles de cambios automáticos.

\section{Tipos de Sistemas KERS}

KERS Mecánico: El KERS mecánico utiliza un volante como el dispositivo de almacenamiento de energía y una transmisión variable para controlar y transferir la energía hacia y desde la línea de transmisión.

KERS eléctrico: El sistema de recuperación de energía cinética eléctrica convierte la energía cinética en energía química para el almacenamiento y se utiliza un sistema generador de motor eléctrico como medio de transferencia y control de la energía.

KERS Hidráulico: Este sistema es particularmente adecuado para la aplicación en la ciudad. El frenado regenerativo en vehículos que utilizan una bomba/motor hidráulico de desplazamiento variable junto con un acumulador hidráulico neumático ha atraído considerable interés durante los últimos años.

KERS Hidroeléctrico: Un sistema hidráulico de sinergia eléctrica (HESS) es un acumulador hidráulico y un sistema de energía híbrida de batería. Este sistema sigue una estructura de energía híbrida para integrar una batería de alta energía específica con un acumulador hidráulico de alta potencia para obtener la salida requerida. Por lo tanto, supera los inconvenientes de una única fuente de almacenamiento de energía tipo KERS [11] [12].

\subsubsection{Bicicletas}

Unvolante de inercia almacena transitoriamentela energía cinética cuando el ciclista frena o ralentiza el movimiento de labicicleta. Esta energía almacenada en el volante se puede reutilizar o recuperar. Considerando que esta energía habitualmente es perdida durante el proceso de frenado la misma puede ayudar a la reconexión de la velocidad y ac- cionamiento mecánico. De esta manera, se puede aumentar la eficiencia energética, e incrementar la velocidad rápidamente (Figura 3) [13].
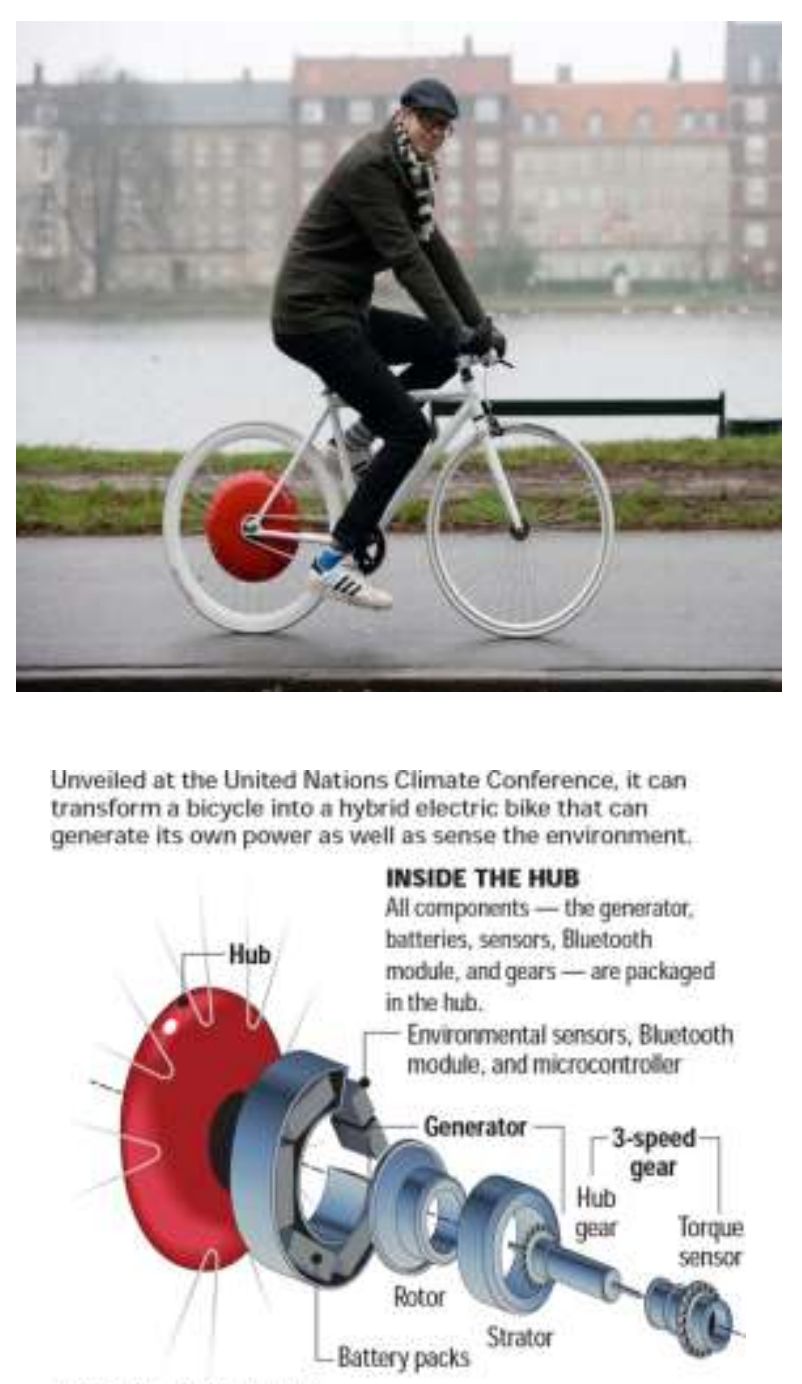

SOUBCE-MITS SESEAble City Lab

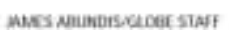

Figura 3. Sistema KERS - Copenhaguen Wheel [13], [14]

La figura (3) muestra la estructura interna utilizada en sistemas KERS para bicicletas. El proyecto desarrollado por el MIT fue denominado Copenhaguen Wheel, puesto que solo se adapta el neumático posterior a cualquier bicicleta y actualmente está siendo introducido en esa ciudad. Varios sensores y módulos ayudan a controlar y almacenar datos de recorrido que facilitan la conexión a aplicaciones móviles. 


\subsection{Transporte pesado}

\subsubsection{Autobuses}

Los primeros autobuses fueron los giro-buses (Gyrobus), desarrollados durante la década de 1940. Bajo el principio de un volante de inercia de acero. El volante pesaba $1500 \mathrm{~kg}$ y tenía un diámetro de 1,6 m. Cada cinco kilómetros, el vehículo se detenía por unos minutos, conectaba su toma a la red y recargaba su impulsor, que accionaba un generador eléctrico. Este impulsor generaba movimiento, con baja resonancia a 3.000 rpm [15].

El alto consumo de energía, una gran necesidad de mantenimiento y reparación y el uso de la carretera debido a la pesadez de los autobuses derivó en la salida de utilización de este tipo de auto buses. Cuando un autobús está desempeñándose en áreas urbanas, genera un gran número de arranques y paradas, haciendo que el volante inercial sea una opción adecuada para el sistema de control de potencia. Los nuevos sistemas Flybrid (Empresa dedicada al desarrollo de volantes para vehículos y buses) reportan un ahorro de combustible del $45 \%$ para un autobús de 17 toneladas métricas en el ciclo de conducción del autobús de Londres [5]. La optimización de los sistemas KERS hacen posible su aplicación en autobuses (Figura 4), adaptado al sistema de transmisión.
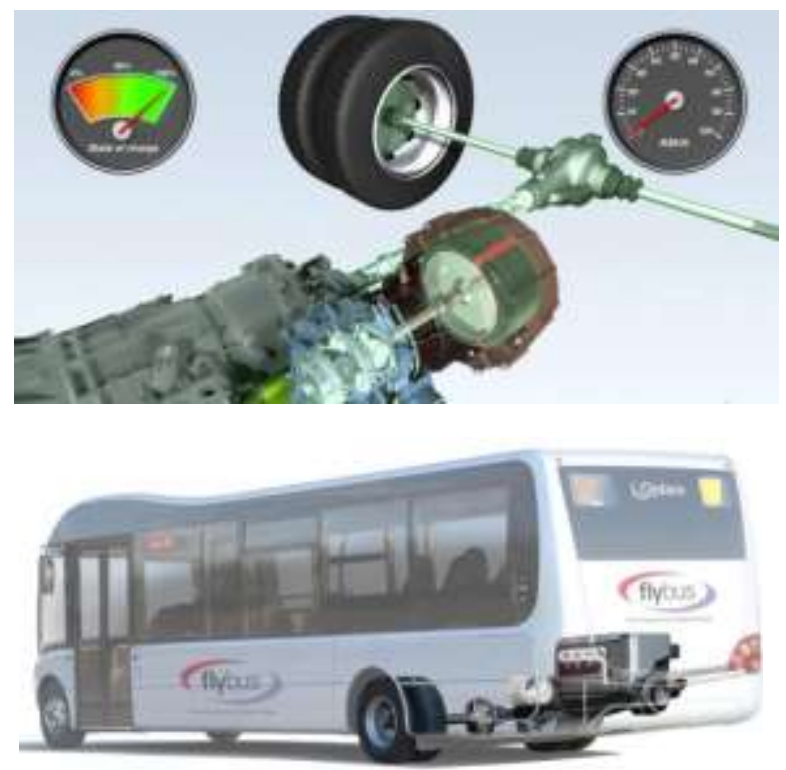

Figura 4. Flybrid para Autobuses[16]
Las características clave del prototipo Flybus, son un volante Ricardo Kinergy como medio de almacenamiento de energía y una transmisión variable continua (CVT) Torotrak que transfiere energía al volante. El volante de inercia Ricardo Kinergy utiliza acoplamiento magnético de bajo costo. El resultado es un sistema hibrido de bajo consumo de combustible y bajas emisiones de carbono.

\subsubsection{Tren de transporte pesado}

Cuando un tren eléctrico frena, la energía es a menudo desperdiciada como calor debido a la falta de un sistema de almacenamiento de energía. Con tal sistema instalado, la energía del freno de un tren se puede utilizar como energía de la aceleración para otro tren.

La Universidad de Texas de Austin probó un volante destinado a la recuperación de energía del freno de tren, que fue diseñado para almacenar $130 \mathrm{kWh}$ a $15.000 \mathrm{rpm}$, y probado hasta 13.600 rpm. El rotor estaba hecho de compuestos de fibra de carbono, y fue suspendido por cojinetes magnéticos activos axial y radialmente con un rotor girado en vacío. Se colocó una máquina eléctrica de 2 MW fuera del vacío (Hedlung, Lundin, De Santiago, \& Abrahamson, 2015). Ricardo (Fabricante de Volantes de Inercia) y Artemis (Sistemas CVT) han diseñado un sistema integrado para trenes eléctricos, que proporciona un incremento del rendimiento en redes ferroviarias eléctricas EMU (Electric Multiple Unit) (Figura 5). Esta configuración puede usar la energía acumulada del volante de inercia (Por medio de un interruptor controlado) o a su vez puede utilizar la red eléctrica para conectarlo al Motor-Generador según convenga para su movimiento. Además, se puede activar y cargar eléctricamente el volante de inercia como muestra la figura.

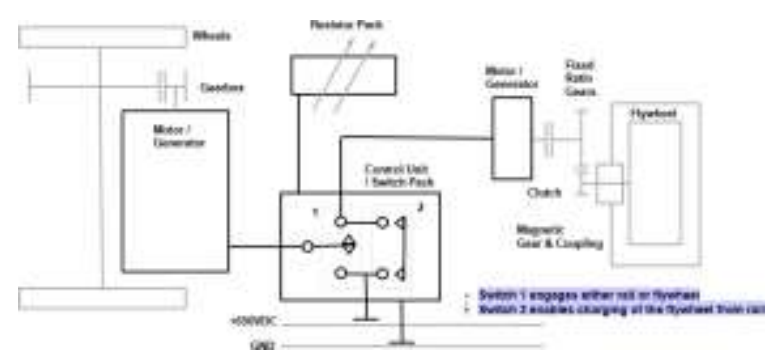

Figura 5. Sistema EMU conectado al volante de inercia [17] 


\subsubsection{Ferrocarril ligero}

La aplicación de la tecnología de volante a la LRT (Light Rail Train) almacena la energía generada durante las paradas de pasajeros frecuentes del tren (Similar a trenes de carga pesada). Los volantes de inercia son capaces de recargar y descargar mucha energía muy rápidamente, por lo que son idóneas para este propósito, las baterías convencionales no duran mucho bajo esas condiciones. Estos sistemas generan ahorros potenciales atractivos para el presupuesto de transporte público de una ciudad. Para el sistema LRT de Edmonton, se ha calculado un ahorro de energía del $31 \%$ y un ahorro de costos del 11\% [5].

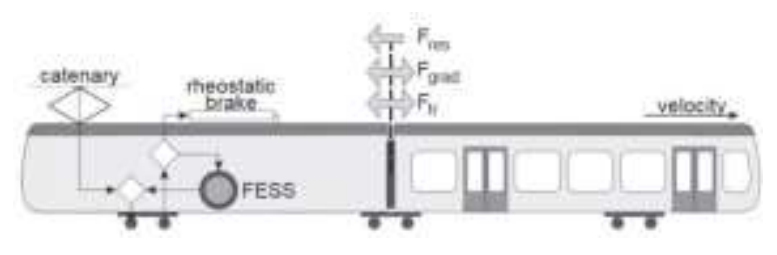

Figura 6. Light Rail Train [18].

\subsubsection{Locomotoras ALPS}

El programa ALPS forma parte del Programa de Alta Velocidad de Nueva Generación, patrocinado por la Federal Railroad Administration. El objetivo del programa ALPS es desarrollar un sistema de propulsión de locomotora de combustibles fósiles capaz de mantener velocidades de $150 \mathrm{mph}$ en la infraestructura existente. El peso de las locomotoras diésel eléctricas convencionales puede crear fuerzas dinámicas que dañan la pista. El motor principal del sistema ALPS es una turbina de gas ligera de alta velocidad que reduce el peso de las locomotoras y las fuerzas dinámicas de la vía a velocidades más altas.

La potencia de propulsión principal es proporcionada por una turbina de gas de 4.000 hp que acciona un alternador síncrono con una potencia nominal de $2 \mathrm{MW}$ con una potencia máxima de 3 MW.La salida del alternador se rectifica para proporcionar energía a un bus de línea, que distribuye la energía a través de la locomotora (Figura 7).

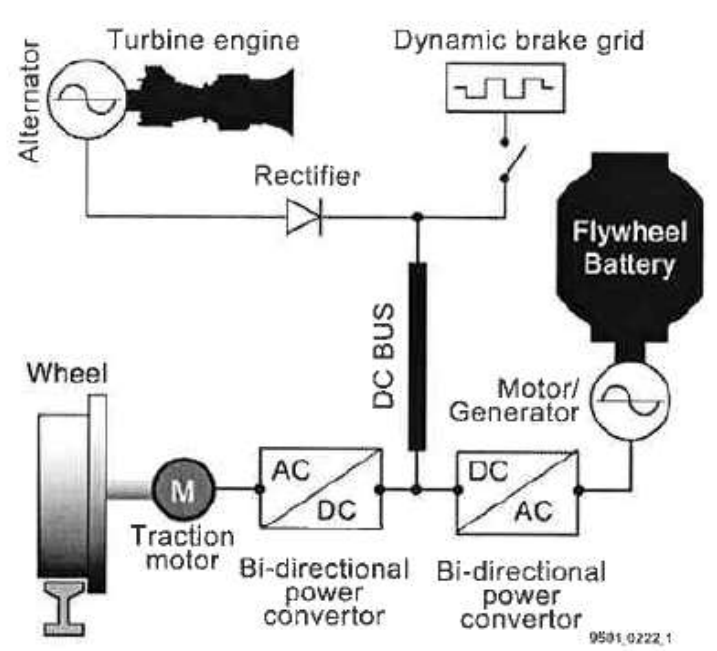

Figura 7. Sistema EMU conectado al volante de inercia [19]

Los motores de tracción AC de la locomotora son accionados a través de variadores de frecuencia conectados al bus DC. El motor/generador del volante también está conectado al bus de salida a través de un convertidor bidireccional de potencia de frecuencia variable. El rotor es levitado en rodamientos magnéticos para minimizar las pérdidas por rotación y permitir el funcionamiento a largo plazo en un ambiente de vacío. Tanto los cojinetes magnéticos radiales como los axiales son de tipo imán permanente con bobinas de control activas montadas en el estator. Esta configuración proporciona un control estable del rotor en un entorno dinámico de locomotora. El volante funciona en un vacío de 0,0131579 atmósferas para minimizar las pérdidas de viento. El rotor del motor está acoplado a un motor/generador de inducción que funciona a presión atmosférica.

El motor/generador de inducción es una máquina de inducción de barra de cobre con una potencia nominal de $2 \mathrm{MW}$ en la gama de velocidades de 7.500 a $15.000 \mathrm{rpm}$. Se conecta a través de un inversor al bus de la locomotora y puede operar en las regiones de motor o de generación, dependiendo de si se ordena acelerar el volante o entregar energía al autobús. El control funciona en un modo $\mathrm{V} / \mathrm{Hz}$ constante (par constante) en el rango de velocidad de 7.500 a $12.000 \mathrm{rpm}$ y en un modo de tensión constante (potencia constante) de 12.000 a $15.000 \mathrm{rpm}$. 


\subsection{Autobús Metro Transit de Houston}

El sistema de autobús de tránsito comparte una filosofía común con la locomotora en que el volante sirve como dispositivo de nivelación de carga. La energía de frenado se utiliza para acelerar el volante y la energía almacenada en el volante se utiliza para complementar la central eléctrica principal para lograr la aceleración o para mantener la velocidad.

El motor/generador es una máquina sincrónica con imanes permanentes montados en el rotor. Los devanados del estator, que están a presión atmosférica, son refrigerados por líquido. El rotor está en vacío, sin embargo, y el calor generado por las corrientes parásitas en cualquier parte del rotor debe ser eliminado por radiación. Se ha dedicado un importante esfuerzo de desarrollo a reducir las pérdidas del rotor, de modo que las pérdidas se puedan disipar sin elevación de temperatura excesiva en el rotor. En la Figura 8 se muestra una imagen de la unidad instalada en el bus [19].

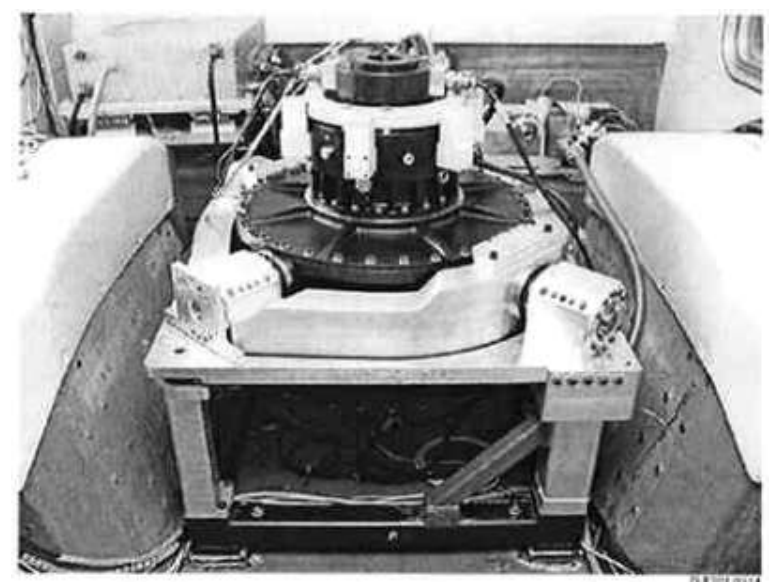

Figura 8. SistemaEMU conectado al volante de inercia [19]

\subsubsection{Sistema DDPM}

Esta tecnología emplea un volante inercial de bobina con un diseño capaz de generar alta velocidad junto con altas cargas de choque (masa de hilado de $21 \mathrm{Kg},>45.000 \mathrm{rpm}$ ). El par se transfiere al volante en una cámara de vacío mediante una forma de acoplamiento magnético a través de la pared de la cámara, sin comprometer la integridad del vacío. La adaptación de velocidad se logra mediante una bomba/motor hidráulico (Pump-Motor) de desplazamiento digital (Digital Displacement) de alta eficiencia (DDPM), en la que los cilindros individuales están controlados por válvulas de solenoide intervenidas digitalmente.

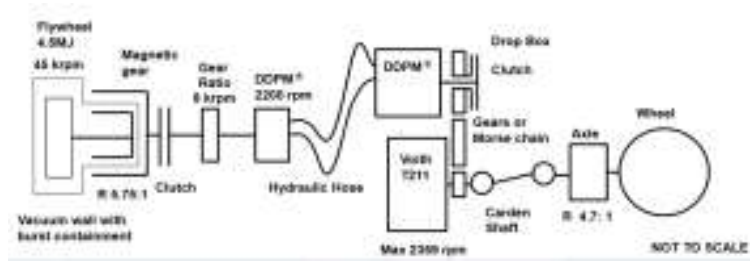

Figura 9. Sistema EMU conectado al volante de inercia [17]

Una aplicación se puede dar conectando a una línea de transmisión DMU (Diesel Multiple Unit). El uso de una conexión hidráulica al módulo de volante permite que este elemento sea montado elásticamente al cuerpo y evite así los altos niveles de vibración asociados con los ejes. Además, la instalación se facilita mediante mangueras flexibles en lugar de ejes o accesorios, lo que permite a los diseñadores utilizar el espacio existente con una interrupción minimizada del equipo existente. Este sistema es válido y adaptable a sistemas EMU [20]. Los dispositivos DDPM se han aplicado con acumuladores hidráulicos como medio de almacenamiento de energía en vehículos híbridos paralelos para autobuses urbanos, para los que se han previsto ahorros superiores al 30\%. La baja densidad de energía de los acumuladores hidráulicos, en comparación con las baterías químicas o cinéticas, las hace inadecuadas para la aplicación en trenes (EMU), por lo tanto, se considera la integración de los volantes de inercia.

\subsection{Tranvías de volante}

Existen dos tipos de tranvías de volante: híbrido y cero-emisiones. Los tranvías del volante híbrido se basan en la energía cinética almacenada en sus volantes para accionar los trenes durante la aceleración y luego recargar los volantes cuando frenan. Los tranvías de volante de emisiones de cero emisiones dependen únicamente de la energía cinética almacenada en sus volantes que se recarga en estaciones y puntos de parada. Estos puntos de parada deben ser relativamente pequeños (inferiores a 0,5 
millas en algunos casos) para que esta tecnología sea viable como un sistema autónomo.

Los sistemas de volante externo también se pueden utilizar en los ferrocarriles electrificados para ayudar a regular el voltaje de la línea a través de la reducción de las demandas de potencia durante la aceleración [21].

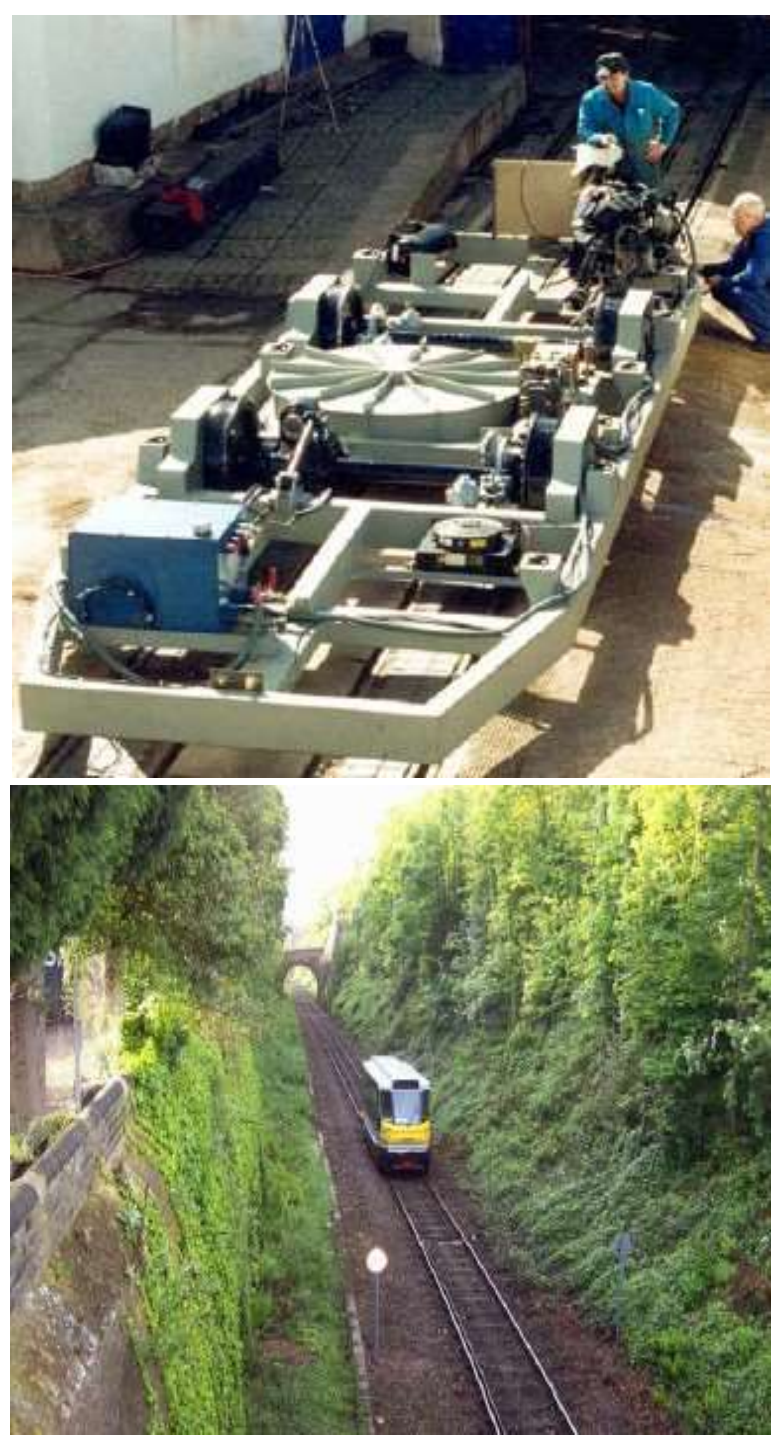

Figura 10. Tranvía de volante [21].

\subsubsection{Tecnología de Levitación}

En 2005, se inició una investigación a gran escala sobre la aplicación de la tecnología ferroviaria de levitación aplicada a ferrocarriles convencionales. Los imanes superconductores se pueden utilizar para levitar, propulsar y guiar objetos pesados (tales como vehículos ferroviarios), permitiéndoles así viajar sin contacto y a alta velocidad. El uso de estos principios condujo a la idea de que el rotor de inercia sea un sistema de almacenamiento de energía de volante, una solución eficaz para la regeneración energética, utilizando bobinas superconductoras en el cojinete del sistema. El sistema Maglev utiliza bobinas superconductoras de alta temperatura REBCO, que generan campos magnéticos de gran alcance, en vez de los imanes permanentes. Con este diseño, la densidad de la fuerza electromagnética aumenta con el cuadrado de la densidad de flujo magnético, lo que permite asegurar suficiente densidad de fuerza electromagnética con menos piezas de material superconductor.

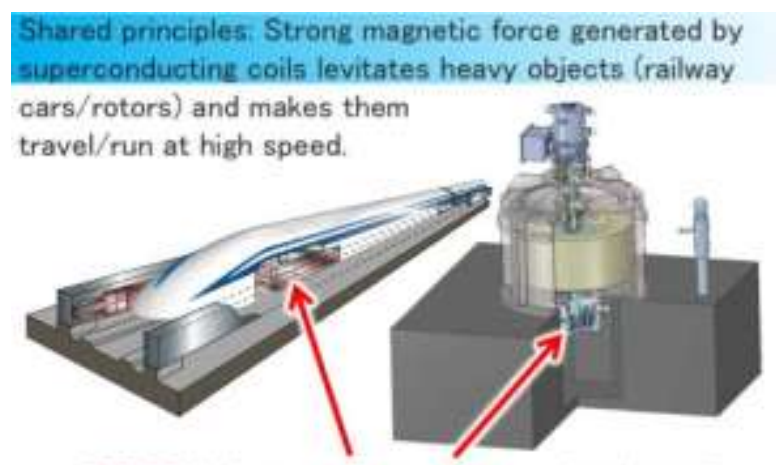

REBCO high-temperature superconducting coils Maglev Flywheel energy storage system Knowledge and experience shared for mutual benefits

Figura 11. Sistema de Levitación Maglev [22].

Principios utilizados en este sistema: Intensa fuerza magnética generada por las bobinas superconductoras levita objetos pesados (vagones/rotores) y los hace viajar y transportarse a alta velocidad [22].

En el tema del volante de inercia, tanto el estator como el rotor del cojinete magnético superconductor deben enfriarse ya que ambos están hechos de material superconductor, por lo que se han hecho esfuerzos investigativos y tecnológicos para mejorar esta tecnología por parte de RTRI. Los mejores resultados se han dado cuando las bobinas superconductoras del estator son refrigeradas por conducción con un refrigerador mientras que las piezas de material a granel superconductoras levitadas son enfriadas por medio de conducción de gas molecular a través de las bobinas (Figura 12). En las últimas investigaciones se utilizaron bobinas superconductoras de alta temperatura de bismuto en lugar de bobinas superconductoras de alta temperatura REBCO. La investigación demostró que es eficaz el enfriamiento con gas de helio 
delgado, así como levitar con éxito y girar el rotor sin contacto. La prueba de carga y velocidad a escala completa de un cojinete magnético superconductor, utilizando bobinas de alambre superconductoras de bismuto a alta temperatura, confirmó una serie de asuntos que se estaban estudiando, incluyendo el tema de si el cojinete era capaz de soportar una carga máxima de 60 kN [22].

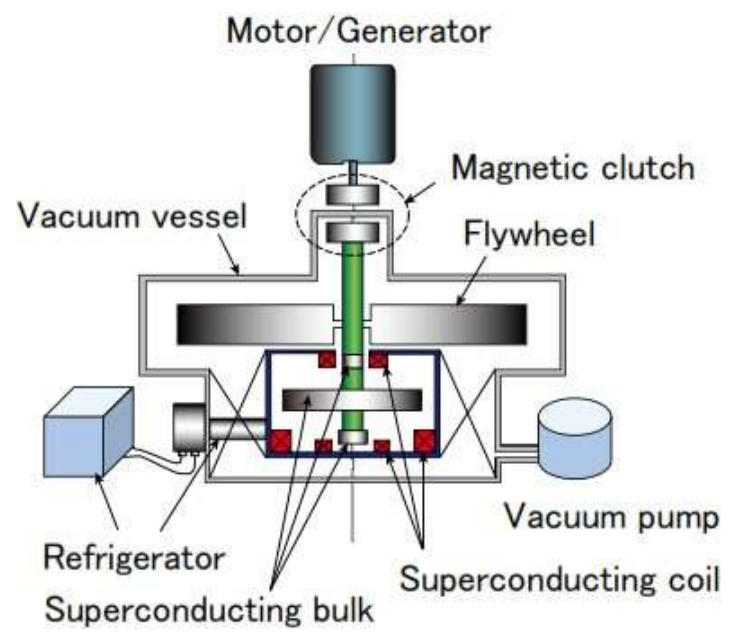

Figura 12. Configuración para Sistema Maglev [22]

Respecto a la tecnología utilizada en varios países (Japón y Alemania), la tecnología Maglev puede tener un mecanismo físico muy diferente, incluyendo la suspensión electromagnética (EMS) y la suspensión electrodinámica (EDS). Mientras que la técnica de EMS (Transrapid alemán) se rige por fuerzas atractivas entre los electroimanes y el carril de acero EDS (Yamanashi japonés Maglev) dependen de fuerzas repulsivas.

En general, la tecnología de volante del maglev se ha utilizado en autos de turismo, trenes, y aviación. En vehículos eléctricos, las baterías de almacenaje del volante del maglev son capaces de acelerar el vehículo en una sola impulsión como una sola energía, y utilizar las baterías del coche eléctrico como energía auxiliar. Cuando se adapta la energía auxiliar, el volante del Maglev estabiliza la salida de energía de las baterías eléctricas y mejora su ciclo de vida. Además, el Maglev mejora la eficiencia de recuperación de energía de frenado regenerativo. Cuando el vehículo eléctrico esta cuesta abajo, deslice o frene, la batería del volante del maglev puede almacenar muy rápidamente la energía mecánica y mejorar la energía de frenado regenerativa [23].

\subsection{Sistema Gimbal parabus}

El objetivo de este sistema es incrementar el potencial de almacenamiento de energía del sistema de volante, reducir los efectos inerciales de los distintos y maximizar el uso de tecnologías de bajo costo. La reducción inercial de los volantes disminuye la carga que experimentan los rodamientos debido a los efectos giroscópicos generados por el desplazamiento del autobús. Normalmente se utilizan cardan para ayudar a manejar estas cargas, pero los cardán aumentan el volumen y el peso del sistema [24].

Eldiseñodel cardán para esta aplicación del volante utiliza dos pares de puntos de pivote para aislar el cardán de los movimientos del bus y mantener el eje de rotación vertical (Figura 13). El cardán permite un giro de $15^{\circ}$ del volante en cualquier dirección para acomodar los movimientos del vehículo. El centro de gravedad del volante está desplazado por debajo de los ejes cardan para inducir una fuerza natural del muelle del péndulo que actúa para mantener el volante vertical.

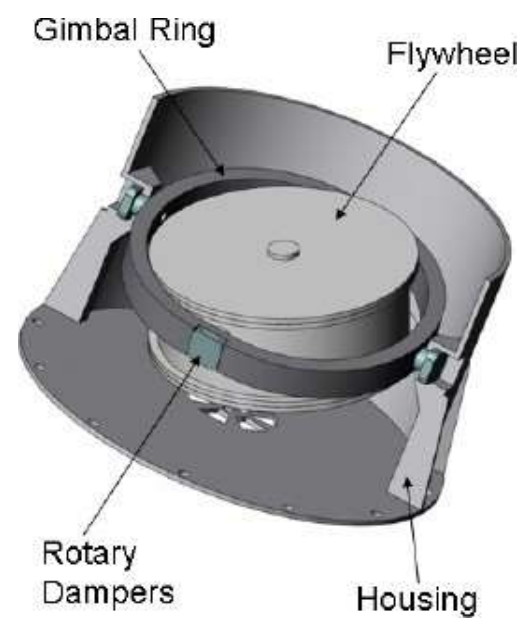

Figura 13. Diseño de sistema Gimbal [24].

El amortiguamiento para el cardán es proporcionado por un conjunto de amortiguadores giratorios en cada eje. El rotor se basa principalmente en el acero para la estructura del volante y se hace hincapié en la reducción de tamaño para las ventajas de envasado del vehículo. Si se compara con los ultracapacitores, estos son capaces de proporcionar algunas de las mismas ventajas que un sistema de almacenamiento de energía del volante. Los ultracapacitores y volantes de inercia en comparación con las baterías químicas, estos tienen mayor densidad de potencia. Con respecto a la capacidad 
de almacenamiento de energía y las densidades de potencia, los ultracapacitores y los volantes son comparables. El único beneficio de los volantes es que no hay degradación del rendimiento a lo largo de la vida útil del volante.

Luego de diez años de vida operativa, los ultracondensadores pueden perder una capacidad del $20 \%$ al $30 \%$ operando a temperatura ambiente. Los límites de tiempo de vida en el sistema de volante se deben principalmente a los cojinetes del elemento rodante para una vida útil de 10 años. Después de 10 años de servicio los cojinetes pueden ser reemplazados para extender la vida de los volantes. Los auxiliares del sistema incluyen inversores y controladores para los generadores de motores, circuito de refrigeración de líquidos y una bomba de vacío. Cada volante con cardán y contención tiene una masa de $390 \mathrm{~kg}$. La masa total del sistema sería de $860 \mathrm{~kg}$ con un volumen de $1 \mathrm{~m} 3$ [24].

Tabla 1. Resumen de sistemas

\begin{tabular}{|c|c|c|c|c|}
\hline Sistema & Tipo & Condición & Rpm & Kwh \\
\hline $\begin{array}{l}\text { Sistema KERS Auto- } \\
\text { móviles }\end{array}$ & $\begin{array}{l}\text { Sistemas: Mecánico, } \\
\text { eléctrico, hidráulico, } \\
\text { hidroeléctrico }\end{array}$ & $\begin{array}{l}\text { Se combina con sistemas } \\
\text { híbridos, CVT, o Supercon- } \\
\text { densadores }\end{array}$ & 60000 & 0.12 \\
\hline $\begin{array}{l}\text { Sistema KERS Bici- } \\
\text { cletas }\end{array}$ & Sistema mecánico & $\begin{array}{l}\text { Sensores inteligentes de } \\
\text { torque }\end{array}$ & - & - \\
\hline $\begin{array}{l}\text { Sistema KERS Auto- } \\
\text { buses }\end{array}$ & $\begin{array}{l}\text { Sistema KERS optimi- } \\
\text { zado }\end{array}$ & $\begin{array}{l}\text { Hibrido con Transmisión } \\
\text { CVT }\end{array}$ & 60000 & 0.11 \\
\hline \multirow{2}{*}{$\begin{array}{l}\text { Tren de transporte } \\
\text { pesado }\end{array}$} & \multirow{2}{*}{$\begin{array}{l}\text { Sistema de Línea Eléc- } \\
\text { trica }\end{array}$} & Transmisión CVT & \multirow[b]{2}{*}{15000} & \multirow[b]{2}{*}{130} \\
\hline & & $\begin{array}{l}\text { Doble Motor generador } \\
\text { eléctrico }\end{array}$ & & \\
\hline Ferrocarril Ligero & $\begin{array}{l}\text { Sistemas: de Línea eléc- } \\
\text { trica; Sistema mecánico }\end{array}$ & $\begin{array}{l}\text { Con volante de inercia In- } \\
\text { corporado }\end{array}$ & 6500 & 1.2 \\
\hline Locomotoras ALPS & $\begin{array}{l}\text { Propulsión: combustible } \\
\text { fósil }\end{array}$ & $\begin{array}{l}\text { Incluye: Turbina de gas, } \\
\text { Motor generador, Freno } \\
\text { dinámico }\end{array}$ & 15000 & 133 \\
\hline $\begin{array}{l}\text { Autobús Metro Tran- } \\
\text { sit de Houston }\end{array}$ & $\begin{array}{l}\text { Sistema Motor/genera- } \\
\text { dor Inducción }\end{array}$ & El Frenado acelera el volante & 40000 & 2 \\
\hline Sistema DDPM & $\begin{array}{l}\text { Sistema Hidráulico di- } \\
\text { gital }\end{array}$ & $\begin{array}{l}\text { Uso de acumuladores hi- } \\
\text { dráulicos para generar mo- } \\
\text { vimiento }\end{array}$ & $>45000$ & - \\
\hline \multirow[t]{2}{*}{ Tranvías de Volante } & Sistemas: hibrido; & \multirow{2}{*}{$\begin{array}{l}\text { Volante de inercia incorpo- } \\
\text { rado en el tranvía }\end{array}$} & \multirow[b]{2}{*}{2600} & \multirow[b]{2}{*}{-} \\
\hline & $\begin{array}{l}\text { Sistemas Cero emisio- } \\
\text { nes (no híbrido) }\end{array}$ & & & \\
\hline $\begin{array}{l}\text { Sistema Levitativo } \\
\text { Maglev }\end{array}$ & $\begin{array}{l}\text { Uso de superconducto- } \\
\text { res HTS }\end{array}$ & $\begin{array}{l}\text { Sistema de refrigeración } \\
\text { avanzado. (Criogénico) }\end{array}$ & 11000 & 5 \\
\hline $\begin{array}{l}\text { Sistema Gimbal para } \\
\text { bus }\end{array}$ & $\begin{array}{l}\text { Reductor de efectos } \\
\text { inerciales }\end{array}$ & $\begin{array}{l}\text { Giro de cardan para incre- } \\
\text { mentar la vida de los coji- } \\
\text { netes. }\end{array}$ & 18000 & 1.87 \\
\hline
\end{tabular}




\section{Materiales}

En la actualidad existen materiales comunes para volantes de inercia, y dependerá de su aplicación y diseño para ser utilizado. Los materiales isotrópicos (Por lo general metales) no requieren de mucha atención en la orientación del material. Los materiales compuestos en cambio requieren de buena orientación particularmente los materiales fibrosos. Cuando las fibras unidireccionales están orientadas circularmente se consigue una gran resistencia a las tensiones tangenciales. A esta orientación, en dirección tangencial, se la denomina fibras a 0 grados y se toma como referencia porque es la dirección natural. Como caso especial, el rotor puede formarse por fibras en la dirección axial y se denominan fibras a 90 grados. [9]
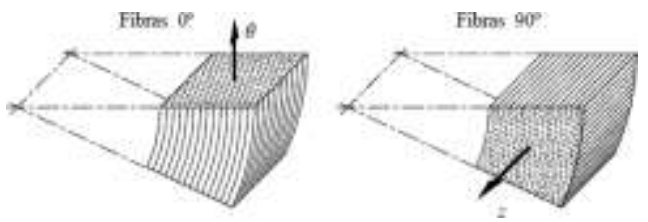

Figura 14. Orientación de las fibras: 0 grados tangencial y 90 grados axial [9].
Si las fibras están orientadas a 0 grados pueden soportar la fuerza centrífuga del rotor a través de la componente tangencial de la tensión. Las fibras a 90 grados no resisten tensiones tangenciales, ni radiales, porque ambas direcciones son normales a esta dirección, sólo resisten tensiones axiales, que aparecen en algunos rotores de gran longitud.

Los rotores compuestos reforzados con fibras orientadas circunferencialmente, probablemente generen grietas circunferenciales, pero son menos propensas a originar fragmentos de proyectiles de vuelo libre en caso de un fallo catastrófico. En general, la geometría y la velocidad del volante determinan la capacidad de almacenamiento de energía [8]. La máxima densidad energética generalmente va de la mano de una excelente resistencia a la tracción junto con una baja densidad de masa. A continuación, en la tabla 2 se puede apreciar esta relación, además que el material que mejor densidad de energía genera tiende a ser el más costoso.

Tabla 2. Datos de diferentes materiales para volantes [8]

\begin{tabular}{lcclc}
\hline Material & $\begin{array}{l}\text { Densidad } \\
\left(\mathbf{k g} \cdot \mathbf{m}^{-3}\right)\end{array}$ & $\begin{array}{l}\text { Resistencia } \\
\text { la Tracción } \\
\mathbf{( M P a )}\end{array}$ & $\begin{array}{l}\text { Densidad de energía máxi- } \\
\text { ma (Para 1 kg) }\end{array}$ & $\begin{array}{l}\text { Costo } \\
\text { Kg }\end{array}$ \\
\hline $\begin{array}{l}\text { Material monolítico } \\
\text { Acero 4340 }\end{array}$ & 7700 & 1520 & $0.19 \mathrm{MJ} / \mathrm{kg}=0.05 \mathrm{kWh} / \mathrm{kg}$ & 1 \\
\hline Compuestos & & & & \\
E-Glass & 2000 & 100 & $0.05 \mathrm{MJ} / \mathrm{kg}=0.014 \mathrm{kWh} / \mathrm{kg}$ & 11.0 \\
S2-Glass & 1920 & 1470 & $0.76 \mathrm{MJ} / \mathrm{kg}=0.21 \mathrm{kWh} / \mathrm{kg}$ & 24.6 \\
Carbono T1000 & 1520 & 1950 & $1.28 \mathrm{MJ} / \mathrm{kg}=0.35 \mathrm{kWh} / \mathrm{kg}$ & 101.8 \\
Carbono AS4C & 1510 & 1650 & $1.1 \mathrm{MJ} / \mathrm{kg}=0.30 \mathrm{kWh} / \mathrm{kg}$ & 31.3 \\
\hline
\end{tabular}

Para la densidad de energía de alta masa, se utilizan materiales de alta resistencia y ligeros, mientras que, para la densidad de energía de alto volumen, se considera la alta resistencia del material. La Tabla 3 muestra las propiedades mecánicas de algunos materiales seleccionados y su capacidad ideal de almacenamiento de energía para un volante en forma de disco. 
Tabla 3. Propiedades mecánicas para diferentes materiales utilizados en volantes [5].

\begin{tabular}{lcccc}
\hline \multicolumn{1}{c}{ Material } & $\begin{array}{c}\boldsymbol{\sigma} \\
\mathbf{( M P a )}\end{array}$ & $\begin{array}{c}\mathbf{Q} \\
\mathbf{( K g / \mathbf { m } ^ { 3 } )}\end{array}$ & $\begin{array}{c}\mathbf{e m} \\
\mathbf{( k J / k g )}\end{array}$ & $\begin{array}{c}\text { ev } \\
\mathbf{( k J / \mathbf { m } ^ { 3 } )}\end{array}$ \\
\hline Acero (AISI 4340) & 1800 & 7800 & 140 & 1092000 \\
Aleación (AlMnMg) & 600 & 2700 & 135 & 364500 \\
Titanio (TiA162r5) & 1200 & 4500 & 162 & 729000 \\
Fibra de vidrio (60\%) & 1600 & 2000 & 485 & 970000 \\
Fibra de carbono (60\%) & 2400 & 1500 & 970 & 1455000 \\
\hline
\end{tabular}

De esta manera y tomando en cuenta la tabla anterior, la mejor opción para diseñar un volante con alta energía y ligero será utilizando fibra de carbono. En cambio, un volante con alta energía y pequeño (en tamaño) tendrá que diseñarse en acero, aunque su peso será elevado. La fibra de carbono tiene un comportamiento muy anisotrópico, con malas propiedades en la dirección radial, por lo que debe tomarse en cuenta estos detalles al considerar los materiales. "Los rotores de acero tienen una energía específica de hasta $5 \mathrm{Wh} / \mathrm{kg}$, mientras que los rotores compuestos de alta velocidad han alcanzado una energía específica de hasta 100 Wh/g" [25]_[5].

Otro ejemplo se da en la tabla 4 en donde se presenta las características mecánicas y económicas de los materiales típicos de acero y compuestos. Aquí, los materiales compuestos como Kevlar, RGlass o E-Glass epoxy son los más indicados para volantes de alta potencia específica y poca masa. El maraging de acero en cambio tiene una buena relación de potencia y volumen por lo que se adapta a espacios limitados [26] [27].

Tabla 4. Propiedades mecánicas y económicas de materiales utilizados en volantes [26], [27].

\begin{tabular}{|c|c|c|c|}
\hline Material & $\begin{array}{l}\text { Densidad } \\
\left(\mathbf{k g} \cdot \mathbf{m}^{-3}\right)\end{array}$ & $\begin{array}{l}\text { Resistencia a la Tracción } \\
\qquad(\mathrm{MPa})\end{array}$ & Costo $€ / \mathrm{Kg}$ \\
\hline 36NiCrMo16 & 7800 & 880 & 6 \\
\hline Maraging 300 & 7800 & 1850 & 32.6 \\
\hline E-Glass Epóxido & 1900 & 1350 & 23.5 \\
\hline R-Glass Epóxico & 1550 & 1380 & 58.0 \\
\hline Kevlar Epóxico & 1370 & 1400 & 72.0 \\
\hline
\end{tabular}

La construcción de un sistema también se puede analizar desde la perspectiva de costo por kWh generado por lo que la fibra de carbono supone una solución de menor costo para almacenar can- tidades relativamente grandes de energía. Algunos costos tienden a bajar acorde a los avances y el movimiento del mercado (Figura 15) [28]. 


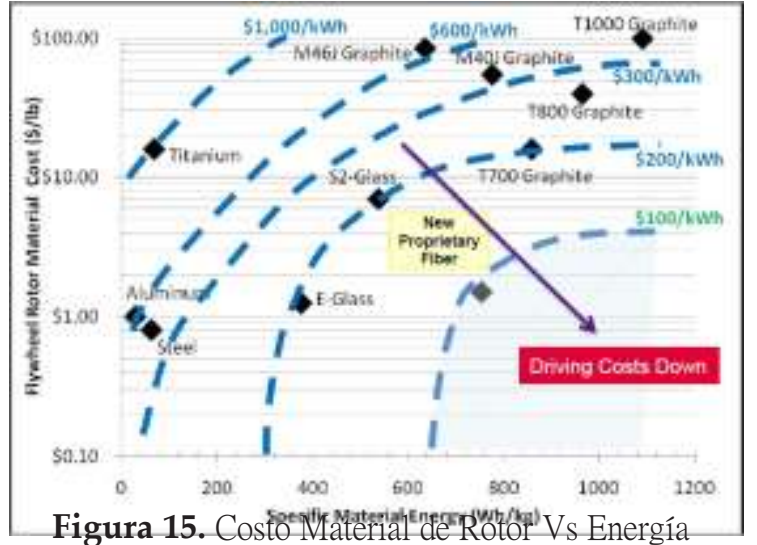

Específica [29]
Existen nuevos materiales que se encuentran en etapas iniciales de uso e investigación, el potencial futuro del almacenamiento de energía del volante se evalúa comparando materiales contemporáneos con algunos nuevos (Tabla 5). Los nanotubos de carbono son poco conocidos pero han recibido interés tanto de la NASA como del Departamento de Defensa de Estados Unidos [2].

Tabla 5. Potencial teórico de volantes con materiales contemporáneos y futuros [2].

\begin{tabular}{lccc}
\hline \multicolumn{1}{c}{ Material } & $\begin{array}{c}\text { Tracción Final } \\
\mathbf{( M P a )}\end{array}$ & $\begin{array}{c}\text { Densidad } \\
\mathbf{( k g . m} \mathbf{3}^{-3}\end{array}$ & $\begin{array}{c}\text { Densidad de } \\
\text { Energía de Ro- } \\
\text { tor (Wh/kg) }\end{array}$ \\
\hline Aluminio 7075 & 572 & 2810 & 28 \\
Acero inoxidable 17-7 PH & 1650 & 7800 & 29 \\
Titanio Ti-15V-3Cr-3Al-3Sn ST 790 ํ & 1380 & 4760 & 40 \\
E-glass (Fibra de vidrio) Advantex & 1400 & 2146 & 90 \\
Compuesto T1000G Toray & 3040 & 1800 & 234 \\
Compuesto T1000G Fibra & 6370 & 1800 & 491 \\
Nanofibras de Carbono cultivadas a vapor & 2920 & 2000 & 202 \\
Nanotubode carbono de pared simple(Bajagama) & 50000 & 1300 & 5341 \\
Nanotubo de carbono de pared simple(Altagama) & 500000 & 1300 & 53418 \\
Nanotubos de carbono multi pared (Baja gama) & 10000 & 1750 & 793 \\
Nanotubos de carbono multi pared (Alta gama) & 60000 & 1750 & 4761 \\
\hline
\end{tabular}

Para el núcleo ferromagnético y el devanado, los materiales de uso común son respectivamente la aleación Fe-Si y el cobre. Los ejemplos de los va- lores de costo para estas materias primas se dan en la tabla 6. Estos valores se obtienen de los datos del fabricante.

Tabla 6. Materiales comúnmente utilizados en núcleo y devanado [26].

\begin{tabular}{llll}
\hline Símbolo & Descripción & Parte del sistema & Valor \\
\hline Ciron & Aleación Fe-Si & PMSM núcleo & $3.0 € / \mathrm{Kg}$ \\
Cco & Cobre & PMSM devanado & $6.0 € / \mathrm{Kg}$ \\
C $_{\text {mag }}$ & Nd-Fe-B & PMSM imán & $140.0 € / \mathrm{Kg}$ \\
\hline
\end{tabular}

Nota: PMSM (Permanent Magnet Synchronous Machine) 
El rotor de un sistema generalmente no supera el $20 \%$ del costo total. En consecuencia, no es válido escalar el coste del sistema del volante a razón de dólares por kilovatio-hora, sin tener en cuenta la composición del coste del sistema del volante.
Sin embargo, el coste incremental por unidad de energía almacenada es calculable para materiales de rotor. La siguiente tabla ofrece una aproximación del costo incremental del material del rotor para aplicaciones de volante de alta velocidad.

Tabla 7. Aproximación del costo incremental acorde al material del rotor [3]

\begin{tabular}{lll}
\hline Material & $\mathbf{\$ ( K w - h )})^{-1}$ & Masa $(\mathbf{K w}-\mathbf{h})^{-\mathbf{1}}$ \\
\hline Compuesto de Carbono & 1200 & 1 \\
Acero 1800 MPa (260000 psi) & 1800 & $7 \mathrm{x}$ \\
Acero 1100 MPa (160000 psi) & 2000 & $12 \mathrm{x}$ \\
Acero 600 MPa (90000 psi) & 4000 & $24 \mathrm{x}$ \\
\hline
\end{tabular}

El motor/generador integrado suele ser de un campo giratorio integrado por electroimanes o imanes permanentes de tierras raras. Las propiedades de los imanes permanentes de alto campo producen densidades de flujo lo suficientemente altas como para permitir que las máquinas con devanados de armadura sin ranura (devanados de entrehierro), no tengan un núcleo de estator magnético. La ausencia de un material ferromagnético en el estator tiene dos impactos importantes en el rendimiento de un motor/generador: En primer lugar, la baja permeabilidad reducirá rápidamente la intensidad del campo magnético cuando se aleja del imán, la tensión inducida se reduce, disminuyendo así la potencia generada. En segundo lugar, no habrá pérdida de calor en el núcleo del estator debido a efectos de histéresis.

La baja resistencia a la tracción de los imanes en comparación con la del compuesto del volante limita su colocación en la proximidad del cubo, así como su número de polos. La Tabla 8 muestra la resistencia a la tracción de materiales magnéticos comunes. Las ferritas debido a su baja conductividad no producen, corrientes parásitas inducidas en la superficie. Sin embargo, algunos de los materiales de tierras raras sinterizados tienen una gran conductividad y por lo tanto sufren de tales problemas [8].

Tabla 8. Propiedades de Materiales magnéticos utilizados en volantes [8]

\begin{tabular}{lccc}
\hline \multicolumn{1}{c}{ Material } & $\begin{array}{c}\text { Densidad } \\
\mathbf{( k g / \mathbf { m } ^ { 3 } )}\end{array}$ & $\begin{array}{c}\text { Resistencia a } \\
\mathbf{l a} \text { Tracción } \\
\mathbf{( M P a )}\end{array}$ & $\begin{array}{c}\text { Remanencia } \\
\mathbf{( T )}\end{array}$ \\
\hline Neodimio-Hierro-Boro Sinterizado (Nd-Fe-B) & $7400-7600$ & 80 & $1.08-1.36$ \\
Samario- Cobalto Sinterizado & $8000-8500$ & 60 & $0.75-1.2$ \\
Ferrita Sinterizada & $4800-5000$ & 9 & $0.2-0.43$ \\
Inyección de material compuesto (Nd-Fe-B) & $4200-5630$ & $35-59$ & $0.40-0.67$ \\
Compuesto Moldeado por Compresión (Nd-Fe-B) & 6000 & 40 & $0.63-0.69$ \\
Inyección de material compuesto - Ferrita & $2420-3840$ & $39-78$ & $0.07-0.30$ \\
\hline
\end{tabular}




\section{Comparativa}

Los sistemas de volante de inercia han desarrollado una tecnología fuerte y pueden competir directamente con sistemas de baterías o pilas de combustible. Los materiales utilizados en volantes de inercia son amigables con el ambiente y tienen larga vida útil. Las aplicaciones son muy variadas y pueden manejar altas velocidades de descarga sin degradación. Las futuras innovaciones dan ventaja a los volantes de inercia respecto a la densidad de energía sobre otras tecnologías recientes (Figura 16).

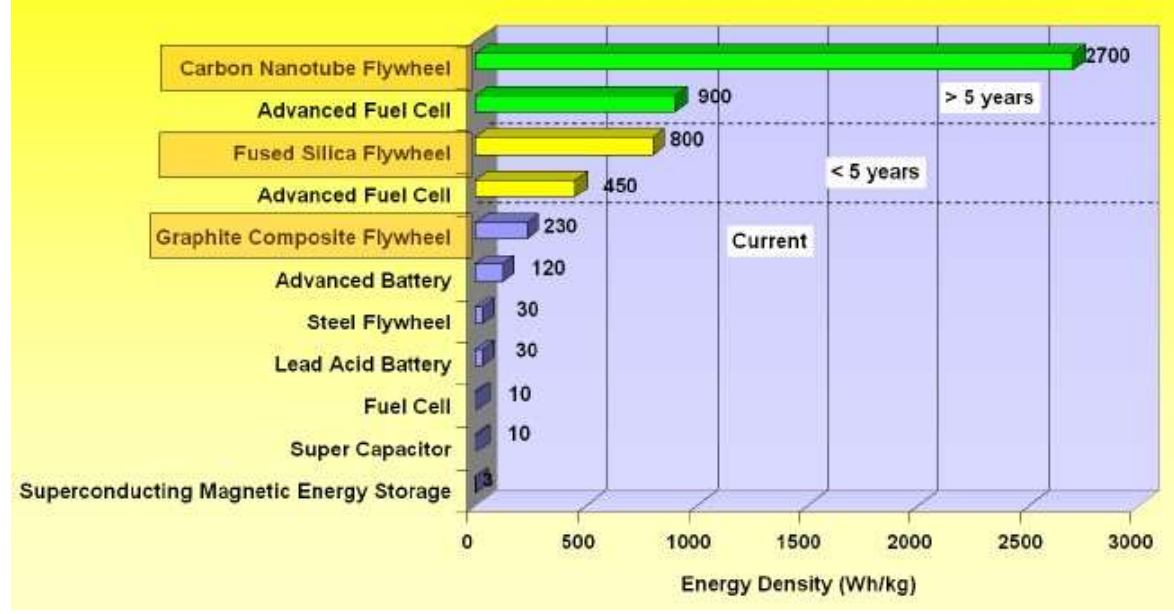

Figura 16. Nuevas aplicaciones de materiales en volantes de inercia [29].

El costo es uno de los factores decisivos en la selección de una tecnología de almacenamiento de energía sobre otra, además del cumplimiento del requerimiento específico de la aplicación. Los volantes deben competir con las baterías y los ultracapacitores sobre la base del costo, aunque el costo se debe evaluar durante la vida útil de un sistema.

La competencia relativa de los costos de los ultracapacitores, baterías y volantes puede presentarse en términos de potencia y tiempo de descarga. La figura 28 muestra las regiones en las que los volantes, los condensadores y las baterías son más rentables. Así como las valoraciones de los fabricantes de sistemas de volante. La región sombreada indica el espacio en donde los volantes presentan ventajas.

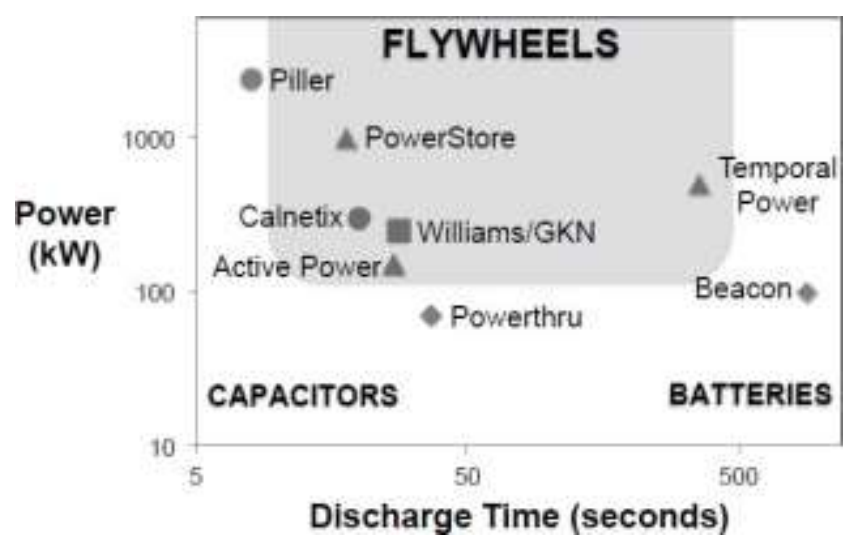

Figura 17. Comparativa entre Ultracapacitores, baterías y volantes [3]. 
Los volantes son convenientes para aplicaciones que requieren energía durante más de varios segundos y hasta varias o decenas de minutos, con un ciclo de vida útil alta. Para aplicaciones que requieren menos de $100 \mathrm{~kW}$, los costos del sistema conducen a que los volantes sean más costosos y menos competitivos.

Comparando con diversas fuentes de energía, se puede considerar que los sistemas FESS (Flywheel
Energy Storage System) tienen una vida útil prolongada, una alta densidad de energía y un rendimiento de potencia máximo. La eficiencia energética fácilmente puede superar un 90\% (Tabla 9). La baja densidad de energía viene a ser un limitante, aunque las últimas investigaciones han demostrado que puede llegar a superarse esos inconvenientes con nuevos materiales tales como nanotubo de carbono y sílice fundida.

Tabla 9. Propiedades de los sistemas de energía [30]

\begin{tabular}{llllll}
\hline \multicolumn{1}{c}{ Tipo } & \multicolumn{1}{c}{ SISTEMAS DE ALMACENAMIENTO DE ENERGÍA } & \\
& $\begin{array}{c}\text { Energética } \\
(\mathbf{\%})\end{array}$ & $\begin{array}{c}\text { Densidad de } \\
\text { Energía } \\
\mathbf{( W h} / \mathbf{K g})\end{array}$ & $\begin{array}{c}\text { Densidad de } \\
\text { Potencia } \\
\mathbf{( W / K g )}\end{array}$ & $\begin{array}{c}\text { Ciclo de vida } \\
\text { (Ciclos) }\end{array}$ & $\begin{array}{c}\text { Auto } \\
\text { Descarga }\end{array}$ \\
\hline Pb-Ácido & $70-80$ & $20-35$ & 25 & $200-2000$ & Baja \\
Ni-Cd & $60-90$ & $40-60$ & $140-180$ & $500-2000$ & Baja \\
Ni-MH & $50-80$ & $60-80$ & 220 & $<3000$ & Alta \\
Li-Ion & $70-85$ & $100-200$ & 360 & $500-2000$ & Media \\
Li-Polymer & 70 & 200 & $250-1000$ & $>1200$ & Media \\
NaS & 70 & 120 & 120 & 2000 & - \\
VRB & 80 & 25 & $80-150$ & $>16000$ & Despreciable \\
EDLC & 95 & $<50$ & 4000 & $>50000$ & Muy Alta \\
Pumped hydro & $65-80$ & 0.3 & - & $>20$ años & Despreciable \\
CAES & $40-50$ & $10-30$ & - & $>20$ años & - \\
Volante (Ace- & 95 & $5-30$ & 1000 & $>20000$ & Muy Alta \\
ro) & & & & & \\
Volante (Com- & 95 & $>50$ & 5000 & $>20000$ & Muy Alta \\
puesto) & & & & & \\
\hline
\end{tabular}

Nota: $\mathrm{VRB}=$ Vanadium Redox Battery CAES= Compressed air energy storage EDLC= Electric double-layer capacitors

\section{Conclusiones}

El sistema de volante de inercia no es una tecnología nueva y su funcionamiento es relativamente simple, y principalmente en los últimos años se han logrado adaptar diseños para múltiples aplicaciones sencillas y avanzadas.

En los sistemas de transportación liviana (automóviles), existen limitantes de densidad de energía por lo que se han considerado aplicaciones en sistemas híbridos aprovechando la energía cinética desperdiciada a través de frenos regenerativos. Los sistemas actuales apuestan por elementos como baterías, volante de inercia y transmisiones CVT.

En transportación pesada (Buses, camiones, trenes, entre otros), existen avances significativos que han dado lugar a la creación de nuevas tecnologías aplicaciónes tales como el uso de los HTS con alta capacidad de velocidad, amortiguación y descarga; sistemas DDPM hidráulicos que son adaptables 
a sistemas que ocupan energías fósiles; sistemas Gimbal cuyo propósito es reducir los costos de salida de volantes de inercia, entre otros.

Los nuevos sistemasutilizados en vehículos tienen excelentes ventajas como pérdidas de fricción muy baja, larga vida útil, poco mantenimiento, costo ambiental reducido, reducción de emisiones contaminantes, entre otros; y son adaptables a cualquier tecnología de transporte.

Los sistemas de alta velocidad y descarga son más costosos, y algunos sistemas necesitan de refrigeración criogénica (HTS). La elevación de los costos viene dada por el tipo de materiales que ocupa el volante de inercia. La velocidad máxima se limita por las propiedades del material. El desarrollo de nuevos materiales y la reducción de costos es un objetivo en el cual trabajan actualmente varias empresas dedicadas a la fabricación de volantes.

Actualmente la tecnología de fibra y cojinetes superconductores ha permitido el desarrollo de sistemas de almacenamiento de energía de alta eficiencia y alta densidad de energía.

Nuevos estudios revelan que la tecnología de FESS puede competir con los ultracondensadores tanto desde el punto de vista del costo como de potencia. [29] Se ha considerado investigar nuevos materiales para volantes como el nanotubo de carbono y combinar con tecnologías HTS, dando como resultado sistemas con alta densidad energética y alta potencia, tiempos de carga y descarga rápidos comparables con las mejores pilas de combustible actuales, ciclos de vida superiores a 25 años y con un punto importante que es el bajo costo y mantenimiento. Algunos de estos sistemas alcanzan velocidades superiores a 500.000 RPM.

\section{Referencias}

[1] D. Schultz, Conventional and Advanced Flywheel Energy Storage, 2014.

[2] M. Hedlung, J. Lundin, J. De Santiago y J. Abrahamson, «Flywheel Energy Storage for Automotive Applications,» Energies, n⿳⺈ 8, pp. 10636-10663, 2015.

[3] D. Bender, «Flywheels,» California, 2015.
[4] F. Martínez, Diseño de un acumulador de energía cinética para sistema StartEStop, 2013.

[5] CIEMAT, «Kinetic energy storage based on Flywheels: Basic concepts, state of the art and analysis of applications,» Project EERA, 2015.

[6] J. D. Sujit Kumar, «Appliaction and Use of Flywheel in Engineering: Overview,» International Journal of Advances in Science Engineering and Technology, pp. 85-90, 2013.

[7] Ricardo, «Breakthrough in Ricardo Kinergy second generation high-speed flywheel technology,» 22 Agosto 2011. [En línea]. Available: http://www.ricardo. com/en-GB/News--Media/Press-releases/News-releases 1/2011/Breakthrough-in-Ricardo-Kinergy-second-generation-high-speed-flywheel-technology/.

[8] B. Bolund, H. Bernhoff y M. Leijon, «Flywheel energy and power storage systems. Renew Sustain Energy,» Renewable and Sustainable Energy Reviews, pp. 235258, 2007.

[9] L. Masferrer, Análisis y Diseño de Volantes de Inercia de Materiales Compuestos, 2005.

[10] A. Sáenz y J. Tresaco, «Sistema para la Recuperación de la Energía Cinética perdida durante la Frenada en la Fórmula 1,» 2010. [En línea]. Available: https://zaguan.unizar.es/record/5722/files/ INPRO--2011-001.pdf.

[11] K. N. Shreemoy, Design of Kinetic Energy Recovery System for Bicycle, 2014.

[12] S. H. Saheb, C. Deepak y A. Sagar, «Analysis and Application of Various Kers (Kinetic Energy Recovery System) On Bicycle Considering Weight Limitations for Maximum Performance,» International Journal of Engineering Research And Management (IJERM), pp. 14-17, 2015. 
[13] M. López, «La buena vida,» 7 Enero 2015. [En línea]. Available: http:// www.labuenavida.cc/2015/01/la-bicicleta-que-aprende-sobre-la-ciudad/\#.WNBIdvk1_IU.

[14] The Boston Globe, «The Copenhagen Wheel,» Enero 2016. [En línea]. Available: http://archive.boston.com/lifestyle/ green/articles/2009/12/16/the_wheel/.

[15] F. Kirbus, «Autoblog.com.ar,» 11 Marzo 2011. [En línea]. Available: http://autoblog.com.ar/2011/03/11/gyrobus-comoera-viajar-en-el-colectivo-con-sistemakers-de-los-anos-\%E2\%80\%9850/.

[16] Torotrack Group, «Bus KERS - Coming to a bus stop near you,» 05 Enero 2015. [En línea]. Available: http://www.torotrak.com/bus-kers-coming-to-a-busstop-near-you/.

[17] J. Wheals, J. Taylor y W. Lanoe, «Rail Hybrid using Flywheel,» de Den Danske Banekonference 2016 - Tivoli Congress Centre, Copenhagen, 2016.

[18] M. Alba, «Engeenering.com,»19 Diciembre 2016. [En línea]. Available: http:// www.engineering.com/DesignerEdge/ DesignerEdgeArticles/ArticleID/13957/ Using-Flywheels-in-Light-Rail-Transit. aspx.

[19] H. Jordan, J. Herbst y R. Hayes, «Flywheel Energy Storage System and their Aplications,» SMMA 2002 Fall Technical Conference, pp. 1-5, 2002.

[20] J. Wheals, W. To, M. Vigar, J. Hodgson, J. .Buchanan, A. Robertson, J.Macpherson, J. Taylor, W. Lanoe y M. Heaton, «Viable flywheel system for rail,» 2015.

[21] Sintropher, «Innovative Technologies for Light Rail and Tram: A European reference resource,» INTERREG IVB, UE, 2015.
[22] K. Nagashima, «Research and Development Concerning Superconducting Maglev and Research on Applying Its Technology to Conventional Railways System,» QR of RTRI, pp. 175-178, 2016.

[23] G. Hui, Z. Changguo, C. Liangliang y L. Huailiang, «Research on maglev flywheel energy storage system for electric vehicle,» Advanced Materials Research, pp. 1078-1085, 2012.

[24] C. Hearn, M. Flynn, M. Lewis, R. Thompson, B. Murphy y R. Longoria, «Low Cost Flywheel Energy Storage for a Fuel Cell Powered Transit Bus,» IEEE - Report, pp. 1-8, 2007.

[25] F. Martinez, Diseño de un acumulador de energía cinética para sistema StartEStop, Catalunya: Universitat Politécnica de Catalunya, 2013.

[26] J.-C. Olivier, N. Bernard, S. Trieste, L. Mendoza y S. Bourguet, «Techno-economic Optimization of Flywheel Storage System in transportation,» Symposium de Genie Electrique, 2014.

[27] M. S. KUMAR y Y. KUMAR , «OPTIMIZATION OF FLYWHEEL MATERIALS USING GENETIC ALGORITHM,» ACTA TECHNICA CORVINIENSIS Bulletin of Engineering, pp. 37-40, 2012.

[28] M. Lazarewicz y D. Bender, «Flywheels: An Overview of Applications and Technology,» Helix power, 2013.

[29] Boeing Research \& Technology, «Boeing Flywheel Energy Storage Technology,» BOEING, 2012.

[30] S. Vazquez, S. Lukic, E. Galvan, L. Franquelo y J. Carrasco, «Energy Storage Systems for Transport and Grid Applications,» IEEE TRANSACTIONS ON INDUSTRIAL ELECTRONICS, pp. 38813895, 2010. 
[31] L. Giorgi, «Sustainable mobility. Challenges, opportunities and conflicts - a social science perspective,» International Social Science Journal, pp. 179-183, 2004.

[32] United Nations Centre for Regional Development, Mobility and NMT in Sustainable Urban Development - Role of City, Ulaanbaatar: UNCRD, 2018.

[33] OECD, Managing the Fundamental Drivers of Transport Demand, París: ECMT Publications, 2003.

[34] OECD, «Towards Green Growth: Monitoring Progress,» OECD, PAris,2011.

[35] G. Santos, H. Behrendt y A. Teytelboym, «Part II: Policy instruments for sustainable road transport,» Research in Transportation Economics, pp. 46-91, 2010.

[36] B. Elzen, F. W. Geels y K. Green, System Innovation and the Transition to Sustainability: Theory, Evidence and Policy, Cheltenham: Edward Elgar Publishing, 2004.

[37] UNEP, Design for Sustainability, País: UNEP, 2006.

[38] H. B. de Barros Pereira y P. F. de Almeida Souza, «Design for Sustainability:» 5th European Academy of Design Conference, pp. 1-17, 2003.

[39] T. Goldman y R. Gorham, «Sustainable urban transport: Four innovative directions,» Technology in Society, pp. 261-273, 2006.

[40] P. Nijkamp, U. B. Erik Verhoef y C. Rodenburg, «Sustainable mobility,» Transportation engineering and planning, pp. 1-10, 2001.

[41] P. Nieuwenhuis, P. Vergragt y P. Wells, The Business of Sustainable Mobility: From Vision to Reality, New York: Routledge, 2017.
[42] W. R. Black, «Socio-economic barriers to sustainable transport,» Journal of Transport Geography, pp. 141-147, 2000.

[43] C. Doll y M. Wietschel, «Externalities of the transport sector and the role of hydrogen in a sustainable transport vision,» Energy Policy, pp. 4069-4078, 2008.

[44] D. Banister, «Cities, mobility and climate change,» Journal of Transport Geography, pp. 1538-1546, 2011.

[45] J. M. López Pol y M. d. V.Segarra-Oña, «Smart Cities: nuevos focos de innovación para un desarrollo sostenible,» Universidad Politécnica de Valencia, pp. 1-29, 2013.

[46] P. Neirotti, A. De Marco, A. C. Cagliano, G. Mangano y F. Scorrano, «Current trends in Smart City initiatives: Some stylised facts,» Cities, pp. 25-36, 2014.

[47] T. Nam y T. A. Pardo, «Conceptualizing Smart City with Dimensions of Technology, People, and Institutions,» The Proceedings of the 12th Annual International Conference on Digital Government Research, pp. 282-291, 2011.

[48] J.Zawieska y P. Jana, «Smart city as a tool for sustainable mobility and transport decarbonisation,» Transport Policy, pp. 3950, 2018.

[49] S. d. Movilidad, «MUNICIPIO DEL DISTRITO METROPOLITANO DE QUITO,» 2016. [En línea]. Available: http:// gobiernoabierto.quito.gob.ec/wp-content/uploads/documentos/pdf/diagnosticomovilidad.pdf.

[50] F. J. Carphio Martínez, «UDLA,» 2015. [En línea]. Available: http://dspace.udla. edu.ec/handle/33000/3656.

[51] M. D. D. M. D. QUITO, «PLAN MAESTRO DE MOVILIDAD,» 2010. [En línea]. Available: http://www.flacsoandes. edu.ec/libros/118765-opac. 
[52] Enerlis; Ernst; Young, «LIBRO BLANCO SMART CITIES,» Septiembre 2012. [En línea]. Available: http://www.innopro.es/ pdfs/libro_blanco_smart_cities.pdf.

[53] D. Cárdenas y F. Camargo, «Las smart cities, la comunicación y el diseño: un reto de la sociedad del conocimiento.,»Virtu@lMente,pp. 42-55,2016.

[54] ANT, «Plan Nacional de Seguridad Vial,» Agencia Nacional de Tránsito,2013.

[55] J. S. Vizcarra, 2010. [En línea]. Available: https://www.puce.edu.ec/documentos/ opsa/Movilidad.pdf.

[56] L. Hora, «La Hora,» 0105 2010. [En línea]. Available: https://lahora.com.ec/ noticia/1028329/pico-y-placa-guc3ada-para-los-carros-que--vienen-a-la-ciudad.

[57] A. M. d. Transito, 2018. [En línea]. Available: http://www.amt.gob.ec/index.php/ pico-placa-homepage.html.

[58] EPMTP, 1503 2017. [En línea]. Available: http://www.trolebus.gob.ec/index.php/ sobre-nosotros/historia-institucional.

[59] M. B. Merizalde, 3011 2017. [En línea]. Available: http://www.elcomercio.com/ actualidad/quito-primer-bus-electrico-transporte.html.

[60] Quitoinforma, 0112 2017. [En línea]. Available: http://www.quitoinforma.gob. ec/2017/12/01/el-primer-bus-articulado-electrico-ya-circula-en-quito/.

[61] A. Almeida, 0112 2017. [En línea]. Available: http://www.extra.ec/actualidad/ bus-electrico-quito-transporte-publico-BG1877892.

[62] BiciQuito, 2017. [En línea]. Available: http://www.biciquito.gob.ec/index.php/ info/que-es.html.
[63] G. M. d. Rumiñahui, 1901 2017. [En línea]. Available: http://www.ruminahui. gob.ec/index.php?option=com_content\&view $=$ article $\& i d=374: \&$ catid $=8 \&$ Ite$\mathrm{mid}=113 \&$ lang $=\mathrm{kw}$.

[64] Distrito Metropolitano de Quito, «Plan maestro de movilidad para el Distrito Metropolitano de Quito: 2009-2025,» Distrito Metropolitano de Quito, Quito, 2009.

[65] RCP Consultores Asociados, «Consultoría a fin de desarrollar los estudios para la armonización del sistema de revisión técnica vehicular obligatoria del Distrito Metropoliano de Quito a la nueva Legislación Nacional y a los requerimientos técnicos actuales,» Agencia Metropolitana de Control de Transporte Terrestre, Tránsito y Seguridad Vial del Municipio del Distrito Metropolitano de Quito, Quito, 2017.

[66] T. Khokhar, «Tres grandes ideas para lograr ciudades y comunidades sostenibles,» 31 Enero 2018. [Enlínea]. Available: http://www.bancomundial.org/es/news/ immersive-story/2018/01/31/3-big-ideas -to-achieve-sustainable-cities-and-communities.

[67] M. Bouskela, M. Casseb, S. Bassi, C. De Luca y M. Facchina, «La ruta hacia las Smart Cities, Migrando de una gestión tradicional a la ciudad inteligente,» 2016. [En línea]. Available: https://publications. iadb.org/bitstream/handle/11319/7743/ La-ruta-hacia-las-smart-cities-Migrando-de-una-gestion-tradicional-a-la-ciudad-inteligente.pdf.

[68] prooV, «Smartphones to Smart Cities: How technology is impacting our lives,» 2017. [En línea]. Available: http://proov. io/2016/08/17/smartphones-to-smart-cities-how-technology-is-imp acting-our-lives/?preview=true. 
[69] S.C.ATLAS, «SMARTCITIES ATLAS,» 0205 2016. [En línea]. Available: http:// www.smartcitiesatlas.com/transporte-y-movilidad-en-una-ciudad-inteligentel.

[70] M. Vicosa, «Elindependiente,» 29042017. [En línea]. Available: https://www.elindependiente.com/economia/2017/04/29/elreto-del-siglo-xxi-es-mejorar-la-calidadde-vida-en-las-ciudades/.

[71] Metro Ecuador, «metroecuador.com,» 0306 2017. [En línea]. Available: https://www.metroecuador.com.ec/ec/ noticias/2017/07/03/municipio-qui- torecogera-datos-planificar-movili- dadsmart-city.html.

[72] Alcalde Metropolitano, «Quito será la primera Smart City de Latinoamérica,» 30 Junio 2017. [En línea]. Available: http:// prensa.quito.gob.ec/index.php?modu$1 \mathrm{l}=$ Noticias \&func $=$ news_user_view\&i$\mathrm{d}=26710 \&$ umt $=$ Quito $\% 20$ ser $\%$ E1\%20 1a\%20primera\%20Smart\%20City\%20 de\%20Latinoam\%E9rica.

[73] EL Comercio, «Movilidad sostenible para una ciudad moderna,» Tendencias, p. 2, 10 Diciembre 2017.

[74] A. Metropolitana, «quito.gob.ec,» 30 06 2017. [En línea]. Available: http:// prensa.quito.gob.ec/index.php?module $=$ Noticias \&func $=$ news_user_view\&i$\mathrm{d}=26710 \&$ umt $=$ Quito $\% 20$ ser $\%$ E1\%20 1a\%20primera\%20Smart\%20City\%20 de\%20Latinoam\%E9rica.

[75] M. A. González, «Criterios de diseño industrial para una propuesta conceptual en el desarrollo de vehículos de los SITP de,» Universidad Nacional de Colombia, Bogotá, 2015.

[76] National Geographic, «Medio Ambiente,» 8 Noviembre 2017. [En línea]. Available: https://www.nationalgeographic.es/ medio-ambiente/2017/07/grandes-proyectos-de-transporte-sostenible-para-luchar-contra-los-atascos-en.
[77] G. M. Martin, «Proceedings of the NCEER/SEAOC/BSSC Workshop on Site Response during Earthquakes and Seismic Code Revisions,» Univ. of Southern Calif, 1994.

[78] ASCE 7, Seismic Evaluation and Retrofit of Existing Buildings, Reston, Virginia: American Society of Civil Engineers, 2016.

[79] R. F. Obrzud y A. Truty, THE HARDENING SOIL MODEL-A PRACTICAL GUIDEBOOK, Préverenges: Structures, 2018.

[80] T. Schanz, P. Vermeer y P. Bonnier, «The hardening soil model: Formulation and verification,» Beyond 2000 in Computational Geotechnics , p. 16, 1998.

[81] PLAXIS, Material Models Manual, 2017.

[82] S. Jaky, «The coefficient of earth pressure in Normally Consolidated Clays,» Precedings of the Seventh European Conference on Soil Mechanics and Foundation Engineering, 1944.

[83] P. Mayne y F. Kulhawy, «Ko-OCR Relationships in soil,» Journal of the Geotechnical Division ASCE Vol108-n6, 851-872, 1982.

[84] J. Biarez y P. Hicher, «Elementary Mechanics of Soil Behaviour. Saturated remoulded soils,» 1994.

[85] Y. Nakamura, «A METHOD FOR ESTIMATING THE DYNAMIC CHARACTERIZATION OF THE SUBSOIL USING MICROTREMORS ON THE SOIL SURFACE,» p. 33, 1989.

[86] Pacific Earthquake Engineering Research Center, «PER Ground Motion Database,» 0406 2019. [En línea]. Available: www. ngawest2.berkeley.edu.

[87] A. Alvarado, Neotectonique et cinematique de la deformation continentae en Equador, Arrete: Universite de Grenoble, 2012. 
[88] R. Aguiar, Espectros de control para la ciudad de Quito-Ecuador (Obtención de espectros mediante modelos determinísticos asociados a las fallas ciegas inversas deQuito), Barcelona: A.H. Barbat, 2015.

[89] H. Parra, Desarrollos Metodológicos y aplicaciones hacia el calculo de la peligrosidad sísmica en el Ecuador continental y estudio e riesgo sísmico en la ciudad de Quito, Madrid: Desarrollos Metodológicos y aplicaciones hacia el calculo de la peligrosidad sísmica en el Ecuador continental y estudio e riesgo sísmico en la ciudad de Quito, 2016.

[90] K. Campbell y Y. Borzognia, NGA-Wets2 Campbell-Bozornia Ground Motion Model for the Horizontal Components of PGA, PGV and 5\% Damped Elastic Pseudo-Acceleration Response Spectra for Periods Ranging from 0.01 to $10 \mathrm{sec}$, Berkeley: Pacific Earthquake Engineering Research Center, 2013.

[91] J. Kaklamanos, L. G. Baise y D. M. Boore, «Estimating Unknown Input Parameters when Implementing the NGA Ground-Motion Prediction Equations in Engineering Practice,» Earthquake Engineering Research Institute, p. 17, 2011.

[92] N. Abrahamson, W. Silva y R. Kamai, «Summary of the ASK14 Ground-Motion Relation for Active Crustal Regions,» Earthquake Spectra, p. 39, 2014.

[93] N.Abrahamson, W.Silviay K.R., Update of the AS08 Ground-Motion Prediction Equation Based on the NGA-West2 Data Set, Berkeley: Pacific Earthquake Engineering Research Center, 2013.

[94] B.Chiouy R. Youngs, Update of the Chiou and Youns NGA Ground Motion Model for Average Horizontal Component of Peak Ground Motion and Response Spectra, Berkeley: Pacific Earthquake Engineering Research Center, 2013.
[95] PEER, Users Manual for the PEER Ground Motion Database Web Application, Pacific Earthquake Engineering Research Center, 2011.

[96] NEC-SE-DS, Norma Ecuatoriana de la Construcción-Capitulo de Peligro Sísmico y Diseño Simoresistente, Quito, 2015.

[97] NEC-SE-DS, Norma Ecuatoriana de la Construcción-Capitulo de Peligro Sísmico y Diseño Simoresistente, Quito, 2015.

[98] NIST GCR 12-917-21, Solil-Structure Interaction for Building Structures, Gaithersburg: U.S Department of Commerce, 2012.

[99] A. Chopra, Dinámica de estructuras, México Df: Pearson, 2014.

[100] NEC-SE-DS, Norma Ecuatoriana de la Construcción-Capitulo de Peligro Sísmico y Diseño Simoresistente, Quito, 2015.

[101] «Instituto Geofísico,» 0210 2019. [En línea]. Available: www.igepn.edu.ec.

[102] MIDAS, New experience Geotechnical analysis System, 2019.

[103] U.S Geological Survey \& Esc. Politécnica Nacional, «Mapa de Fallas y Pliegues Cuarternarias de Ecuador y Regiones Oceanicas Adyacentes,» USGS, Denver \& Quito, 2003.

[104] P. Von Soos, «rundbataschenbuch. Part 4. Ernst \& Sohn,» 1991.

[105] Pacific Earthquake Engineering Research Center, «PER Ground Motion Database,» 2509 2019. [En línea]. Available: www. ngawest2.berkeley.edu.

[106] E. Carreño, «Registros y tratamiento de acelerogramas,» Física y tierra, p. 31, 1999.

[107] NEC-SE-DS, Norma Ecuatoriana de la Construcción-Capitulo de Peligro Sísmico y Diseño Simoresistente, Quito, 2015. 
[108] ISTAS, Enero 2017. [En línea]. Available: http://www.istas.net/web/index.asp?idpagina $=2207$.

[109] G. Martinez y B. Rodriguez, Enero 2012. [En línea]. Available: http://www.eoi.es/ blogs/galirmartinez-barros/.

[110] Habitat, Septiembre 2013. [En línea]. Available: http://habitat.aq.upm.es/boletin/n34/arcor 3.html.

[111] R. Rivera, «Cueronet,» 2014. [En línea]. Available: http://www.cueronet.com/tecnica/normasiso14000_anexo3.htm.

[112] I. Hadjipaschalis, A. Poullikkas y V. Efthimiou, «Overview of current and future energy storage technologies for electric power applications,» Renewable and Sustainable Energy Reviews, nํ13, p. 1513 1522, 2009.

[113] L. Milla, «Evolución de la Energía Convencional y No Convencional,» Industrial Data, vol. 5, nํㅡ 2, pp. 78-85, Mayo 2002.

[114] Red Eléctrica de España, Octubre 2013. [En línea]. Available: http://www.ree.es/ es/red21/idi/proyectos-idi/proyecto-volante-inercia.

[115] RED Eléctrica de España, «Estabilizador de frecuencia y tensión basado en el volante de inercia. Proyecto de I+D+i,» España, 2014.

[116] The Open Door Web Site, «Factors Which Determine the Moment of Inertia of a Body,» 2016. [En línea]. Available: http://www.saburchill.com/physics/ chapters/0022.html.

[117] B. Wheeler, «Flywheel Energy Storage,» 24 Octubre 2010. [En línea]. Available: http://large.stanford.edu/courses/2010/ ph240/wheeler1/.

[118] M. Molina, «Dynamic Modelling and Control Design of Advanced Energy Storage for Power System Applications,» InTech, 2010.
[119] O. Martínez, «UVaDOC Repositorio Documental de la Universidad de Valladolid,» Enero 2014. [En línea]. Available: https://uvadoc.uva.es/bitstream/10324/11605/1/TFM-I-179.pdf.

[120] U.S. Department of Energy, Energy Efficiency and Renewable Energy, «Flywheel Energy Storage,» Federal Energy Management Program, Illinois, 2003.

[121] E. Shahat, A. Keyhani y H. Shewy, «SPACECRAFT FLYWHEEL HIGH SPEED PM SYNCHRONOUS MOTOR DESIGN (CLASSICAL\&GENETIC),» Journal of Theoretical and Applied Information Technology, pp. 83-100, 2005.

[122] Ibertrónica, «Sistemas Ibertrónica,» 2014. [En línea]. Available: http://www.ibertronica.es/blog/tutoriales/funcion-pwm/.

[123] Kinetic Traction Systems, «KTSi (UK) Design Centre Limited,» 2016. [En línea]. Available: http://kinetictraction.com/ applications/.

[124] Caterpillar, «Cat - UPS Flywheel,» Enero 2017. [En línea]. Available: https:// www.cat.com/en_US/power-systems/ electric-power-generation/ups-flywheel. html.

[125] Vycon, «Critical Power Systems,» 2016. [En línea]. Available: http://www.criticalpower.ca/media/uploads/files/overview-Energy-Storage.pdf.

[126] 马同金, «Aircraft carrier energy accumulation flywheel transmission arm return system». China Patente CN103910072 A, 2014.

[127] Bleck \& Bleck Architects, «What is a Flywheel Launched Coaster?,» Bleck \& Bleck Architects, LLC, Chicago, 2015.

[128] P. Philip, «Quora,» 8 Agosto 2014. [En línea]. Available: https://www.quora.com/ How-is-Flywheel-used-in-spacecraft-navigation-and-guidance-system. 
[129] SZYKANA , «KERS Comes to Cars as Jaguar Tests Flywheel Hybrid,» 2 Noviembre 2010. [En línea]. Aailable: https:// szykana.wordpress.com/t ag/kers/.

[130] C. Gable y S. Gable, «Tho ught Co.,» 4 Febrero 2017. [En línea]. Ar www.thoughtco.com/how ailable: https:// rative-braking-work-8546 -does-regene5.

[131] C. Lampton, «Regenera 2016. [En línea]. Availa howstuffworks.com/aut tive Braking,» ole: http://auto. brake-types/regenerative- s-parts/brakes/

[132] STORNETIC, «STORNE TriC Gmb hrim 7 Septiembre 2016. [En línea]. Available: http://stornetic.com/ass ats/downloads/ EN_Pressemitteilung_In.otrans_final. pdf.

[133] F. N. Werfel, R. Rothfeld, T.Riedel, D.Wippich, P. Schirrmeister y R. Koenig, «HTS Bulk Magnetic Applicati on in Flywheel Energy Storage Systems FESS and MAGLEV Transportation,» Adelwitz Technologiezentrum GmbH(ATZ), pp. 1-16, 2014.
[134] PROPEL STEPS - EDUCATION IS EVERYTHING, 23 Abril 2015. [En línea]. Available: https://propelsteps.wordpress. com/2015/04/23/know-how-maglev-trains-works-without-wheels/how-maglev-train-works/.

[135] F. Werfel, U. Floegel-Delor, R. Rothfeld, T. Riedel, B. Goebel, D. Wippich y P. Schirrmeister, «Superconductor bearings, flywheels and transportation,» SUPERCONDUCTOR SCIENCE AND TECHNOLOGY, pp. 1-16,2011. 